\title{
Critical role of Th17 responses in a murine model of Neisseria gonorrhoeae genital infection
}

\author{
B Feinen ${ }^{1}$, AE Jerse ${ }^{2}$, SL Gaffen ${ }^{3}$ and MW Russell ${ }^{1,4}$
}

Host immune responses, including the characteristic influx of neutrophils, against Neisseria gonorrhoeae are poorly understood; adaptive immunity is minimal and non-protective. We hypothesize that $N$. gonorrhoeae selectively elicits Th17-dependent responses, which trigger innate defense mechanisms, including neutrophils and antimicrobial proteins, that it can resist. We found that $N$. gonorrhoeae induced the production of interleukin-17 (IL-17) in mouse T-cells and Th17-inducing cytokines in mouse and human APCs in vitro. IL-17 was induced in the iliac lymph nodes in vivo in a female mouse model of genital tract gonococcal infection. Antibody blockade of IL-17 or deletion of the major IL-17 receptor (IL-17R) in IL-17RA ${ }^{\mathrm{KO}}$ mice led to prolonged infection and diminished neutrophil influx. Genital tract tissue from IL-17RA ${ }^{\mathrm{KO}}$ mice showed reduced production of neutrophil-attractant chemokines in response to culture with N. gonorrhoeae. These results imply a crucial role for IL-17 and Th17 cells in the immune response to N. gonorrhoeae.

\section{INTRODUCTION}

The discovery of Th17 cells as a distinct subset of $\mathrm{CD} 4^{+} \mathrm{T}$-cells has revolutionized our thinking about the relationship between innate and adaptive immunity. By producing interleukin-17 (IL-17) and other cytokines, Th17 cells function as a bridge between these two arms of the immune system. ${ }^{1,2}$ IL-17 can be either protective or pathogenic, as elevated IL-17 has been found in autoimmune diseases such as rheumatoid arthritis and multiple sclerosis, ${ }^{3,4}$ whereas Th17 cells have been shown to exert protection against a variety of bacteria, including Klebsiella pneumoniae, Porphyromonas gingivalis, Escherichia coli, Bordetella pertussis, Staphylococcus aureus, and Mycobacterium tuberculosis, as well as fungi and even viruses. ${ }^{5-10}$ Development of Th17 cells and the ensuing production of IL-17 occur earlier in infection than development of traditional Th1 and Th2 cells, ${ }^{1,2}$ making them critical for bridging the innate and adaptive immune response. ${ }^{11}$

Differentiation and proliferation of Th17 cells is mediated by IL-6, transforming growth factor- $\beta$, IL- $1 \beta$, and IL-23 (references Korn et al..$^{12}$ and Ouyang et al. ${ }^{13}$ ), which are produced by antigenpresenting cells (APCs) in response to microbial infection, following recognition of pathogen-associated molecular patterns. ${ }^{14,15}$ In addition to its role in the development and main- tenance of Th17 cells, IL-23 plays an independent and essential role in the defense against some pathogens by stimulating the production of other factors such as IL-22 (reference Schultz et al. ${ }^{16}$ ). The IL-17 receptor-A (IL-17RA) is widely distributed on endothelial and epithelial cells, osteoblasts, and fibroblasts, and its ligation induces the expression of neutrophil-recruiting chemokines such as KC (CXCL1), LIX (CXCL5), and macrophage inflammatory protein-2 $\alpha$ (MIP-2 $\alpha$ ) (CXCL2) in mice or IL-8 in humans, as well as granulocyte-colony-stimulating factor. ${ }^{9,17-20}$ In extracellular infections where neutrophils are important in host defense, including K. pneumoniae, P. gingivalis, and Candida albicans, deficiency of IL-17RA leads to reduction of chemokine levels and neutrophil recruitment in infected tissues. ${ }^{9,10,21,22}$ In addition to neutrophil recruitment, IL-17, along with IL-22, plays other roles in immune defense, including production of soluble defense factors, such as $\beta$-defensins, lipocalin-2, and S100 proteins, by epithelial cells. ${ }^{23}$

Gonorrhea is a sexually transmitted infection of widespread abundance throughout the world: the World Health Organization estimates that $>60$ million new infections occur annually. ${ }^{24}$ The Centers for Disease Control and Prevention report $>300000$ cases annually in the USA, ${ }^{25}$ and it may be re-emerging in Europe. No vaccine is available to control the

${ }^{1}$ Department of Microbiology and Immunology, Witebsky Center for Microbial Pathogenesis and Immunology, University at Buffalo, Buffalo, New York, USA. ${ }^{2}$ Department of Microbiology and Immunology, Uniformed Services University of the Health Sciences, Bethesda, Maryland, USA. ${ }^{3}$ Division of Rheumatology and Clinical Immunology, University of Pittsburgh, Pittsburgh, Pennsylvania, USA. ${ }^{4}$ Department of Oral Biology, Witebsky Center for Microbial Pathogenesis and Immunology, University at Buffalo, Buffalo, New York, USA. Correspondence: B Feinen (Feinen@buffalo.edu) or MW Russell (russellm@buffalo.edu). 
disease and the continuing development of resistance to antibiotics causes concern that treatment options could become limited. Gonorrhea typically presents as an acute purulent genital tract infection caused by Neisseria gonorrhoeae, and is characterized by the presence of Gram-negative diplococci associated with neutrophils in the exudate. Despite the evident inflammatory response, it is well known that gonorrhea does not induce a state of protective immunity against repeat infection. ${ }^{26-28}$ $N$. gonorrhoeae induces several proinflammatory cytokines, including IL-6, IL- $1 \beta$, and tumor necrosis factor- $\alpha$ (TNF $\alpha$ ), which have been implicated in Th17 responses, but not IL-12 or interferon- $\gamma($ IFN- $\gamma) .^{28-32}$ At present, the mechanisms that govern host responses to $N$. gonorrhoeae, including the neutrophil influx, are not well understood.

We propose that Th17 cells play a significant role in the immune response to $N$. gonorrhoeae through recruitment of neutrophils and other innate defense factors. We have tested this hypothesis in a mouse model of $N$. gonorrhoeae genital tract infection. Our results indicate that $N$. gonorrhoeae induces IL-17 production in vitro and in vivo, leading to IL-17-dependent secretion of IL-6, LIX, and MIP-2 $\alpha$ from genital tract tissues. Furthermore, blocking IL-17A with antibody, or deletion of IL-17RA, in mice prolongs the course of infection with $N$. gonorrhoeae and delays the recruitment of neutrophils.

\section{RESULTS}

\section{Production of cytokines in response to $\mathbf{N}$. gonorrhoeae}

To determine whether $N$. gonorrhoeae is capable of inducing cytokines typical of a Th17 response, we incubated mouse splenic mononuclear cells with either $N$. gonorrhoeae or gonococcal outer membrane vesicles (OMVs) and tested the supernatants for secreted cytokines. After 3 days, the cells produced IL-17 in response to either $N$. gonorrhoeae FA1090 or its outer membranes, as well as the mitogen concanavalin-A (ConA) (Figure 1a, b). IL-17 production increased with time of incubation and dose of OMVs, through 5 days with $5 \mu \mathrm{g} \mathrm{ml}^{-1}$ of OMVs; no significant increase in IL-17 occurred at higher OMV concentrations. Similar results were obtained with $N$. gonorrhoeae strain MS11, and with an Opa-protein deletion mutant of strain FA1090 (reference Jerse et al. ${ }^{33}$; data not shown). Heat treatment of OMV preparations $\left(100^{\circ} \mathrm{C}\right.$ for $10 \mathrm{~min}$ ) did not abrogate the induction of IL-17 (data not shown), suggesting that the stimulatory components were heat-stable. Therefore, to determine whether lipooligosaccharide (LOS) was responsible for inducing IL-17, LOS from $N$. gonorrhoeae strain PID2 and corresponding gonococci were cultured with spleen cells from $\mathrm{C} 3 \mathrm{H} / \mathrm{HeJ}$ ((toll-like receptor 4) TLR4-deficient), C3H/FeJ (TLR4-normal), or TLR2-knockout mice. Gonococcal LOS induced IL-17 production in TLR4-normal (and TLR2-deficient) cells, but not in TLR4-deficient cells (Figure 1c). Furthermore the IL-17 response to gonococci or OMVs was diminished (but not completely abrogated) in TLR4deficient cells, whereas TLR2-knockout cells were responsive to gonococci or OMVs (Figure 1c).

In addition, supernatants from spleen cells cultured with gonococci or OMVs showed production of IL-22 and IL-6, but lack of IFN- $\gamma$ (Figure 1d), consistent with the development of a
Th17 response. Production of IL-12, a hallmark of Th1 response, was not observed at any time point in response to gonococcal stimulation. The ability of $N$. gonorrhoeae to induce the secretion of IL-17, IL-22, and IL-6, but not IFN- $\gamma$, in mouse spleen cell cultures suggests that it is capable of eliciting Th17 responses. As determined by flow cytometry, some of the cells that produced IL-17 were $\gamma \delta$ rather than $\alpha \beta$ T-cells (Figure 1e).

However, IL-23 was not detected in cultures of spleen mononuclear cells stimulated with gonococci or ConA. It is possible that IL-23 was produced but not detected because of rapid uptake by T-cells. Therefore, we determined whether gonococci could selectively induce IL-23 production by APCs alone. When mouse bone marrow-derived dendritic cells (BMDCs) were incubated with $N$. gonorrhoeae or its OMVs for $24 \mathrm{~h}$, IL-6 and IL-23 were detected in the supernatants, whereas IL-12 production was produced only in control cultures stimulated with ConA (Figure 2). Therefore, we conclude that N. gonorrhoeae preferentially stimulates DCs to secrete IL-23, which in turn promotes the differentiation of Th17 cells, but not IL-12, which promotes Th1.

\section{Production of Th17-associated cytokines by human THP-1 cells}

In order to determine whether $N$. gonorrhoeae is capable of inducing cytokines that lead to a Th17 response in human cells, we incubated human THP-1 macrophage-like cells with $N$. gonorrhoeae cells, gonococcal OMVs, or E. coli lipopolysaccharide. Within 1 day, differentiated THP-1 cells produced the proinflammatory cytokines IL- $6, \mathrm{TNF} \alpha$, IL- $1 \beta$, and IL-23 (Figure 3), similar to the results obtained with mouse splenocytes or BMDCs. Likewise, production of IL-12 was not observed in response to N. gonorrhoeae, although it was detected in response to E. coli lipopolysaccharide. Accordingly, human APCs produce Th17- rather than Th1-biasing cytokines in a similar manner to mouse cells.

\section{Role of IL-17 in murine vaginal gonococcal infection}

To determine whether IL-17 is generated in vivo in response to $N$. gonorrhoeae, we employed a previously described mouse genital tract gonococcal infection model. ${ }^{34}$ Mice were treated with estradiol and antibiotics, challenged with live gonococci, and the course of infection was monitored by vaginal culture. Two groups of control mice were included: estradiol- and antibiotic-treated mice, which were sham-infected with vehicle only, and unmanipulated control mice. Draining iliac lymph nodes were removed (from separate groups of mice) starting 1 day after infection and continued for 5 days; the cells were cultured overnight and IL-17 was measured in the supernatants. Starting 2 days after infection, IL-17 production was observed, with production peaking after 3 days and continuing for the duration of infection (Figure 4). Thus mice produce IL-17 in vivo when infected with N. gonorrhoeae.

To determine whether IL-17 plays a significant role in this infection model, BALB/c mice were treated with anti-IL-17blocking antibody before and during infection, while control mice were similarly treated with rat IgG. All mice were treated 


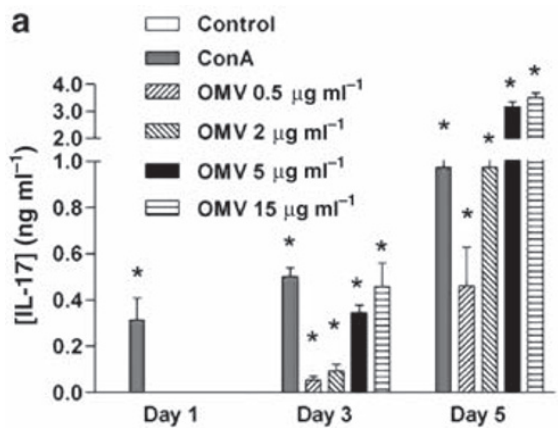

C
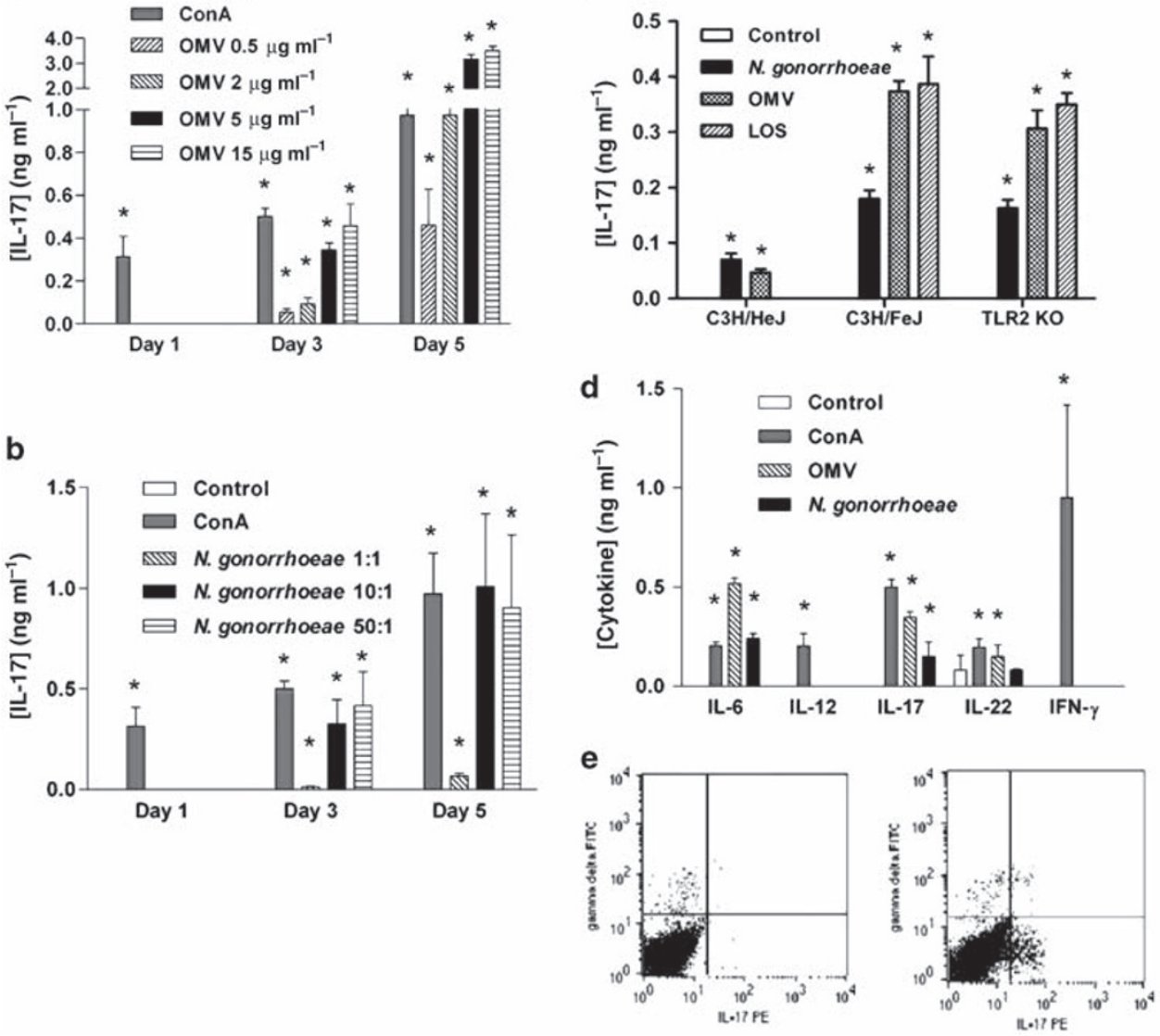

Figure 1 N. gonorrhoeae induces Th17-associated cytokines, but not Th1-associated cytokines. (a) Production of IL-17 from mouse splenic mononuclear cells, incubated in medium only (control) or with $2 \mu \mathrm{g} \mathrm{ml}^{-1}$ ConA or $N$. gonorrhoeae OMVs at various concentrations for 1, 3, or 5 days. Supernatants were assayed for IL-17 by ELISA. (b) Production of IL-17 from mouse splenic mononuclear cells, incubated in medium only (control) or with $2 \mu \mathrm{gml}^{-1}$ ConA or $N$. gonorrhoeae at various MOls for 1,3 , or 5 days. Supernatants were assayed for IL-17 by ELISA. (c) Production of IL-17 from $\mathrm{C} 3 \mathrm{H} / \mathrm{HeJ}$ (TLR4-deficient), $\mathrm{C} 3 \mathrm{H} / \mathrm{FeJ}$ (TLR4-normal), or TLR2-knockout mouse splenic mononuclear cells incubated for 3 days in medium only (control), or with $N$. gonorrhoeae PID2 at MOI 10:1, or with OMVs $\left(5 \mu \mathrm{g} \mathrm{ml}^{-1}\right)$, or LOS $\left(5 \mu \mathrm{g} \mathrm{ml}^{-1}\right)$. Supernatants were assayed for IL-17 by ELISA. (d) Production of IL-6, IL-12, IL-17, IL-22, and IFN- $\gamma$ from mouse splenic mononuclear cells incubated for 3 days in medium only (control) or with $2 \mu \mathrm{g} \mathrm{ml}{ }^{-1}$ or $5 \mathrm{~g} \mathrm{ml}^{-1}$ of OMVs, or $\mathrm{N}$. gonorrhoeae at an MOI of 10:1. Supernatants were assayed for cytokines by ELISA. All experiments (a-d) were performed in triplicate and results are shown as mean \pm s.d.; * indicates cytokine secretion significantly above control levels $(P<0.01$; Student's $t$-test). (e) Flow cytometry profiles of murine spleen cells cultured for 3 days with gonococcal OMVs (right panel), as compared with control unstimulated cells (left panel). Cells were stained for intracellular IL-17 (PE) and surface $\gamma \delta$-T-cell receptor (FITC). ConA, concanavalin-A; IFN, interferon; IL, interleukin; MOI, multiplicity of infection; OMV, outer membrane vesicle.

with estradiol and antibiotics, and challenged with live gonococci as described under section Methods. The course of infection was monitored by vaginal culture and neutrophil influx was determined by microscopic examination of vaginal swabs. Three days after infection, mice treated with the isotype control Ig showed diminished numbers of recoverable $N$. gonorrhoeae. In contrast, mice treated with anti-IL-17 antibody exhibited significantly prolonged infection $(P<0.001)$ and higher recoverable bacterial loads after day $3(P<0.05$; Figure $5 \mathbf{a}, \mathbf{b})$. Neutrophil influx into the genital tracts, determined relative to the epithelial cells in vaginal smears, began on day 3 in control mice, but was delayed until day 4-5 in IL-17 antibody-treated mice and remained at a lower level throughout the duration of the experiment (Figure 5c). These results show that blockade of IL-17 during $N$. gonorrhoeae infection inhibits neutrophil recruitment and enhances the course of infection in this mouse model.
To confirm the importance of signaling through the IL-17R in the response to vaginal infection with $N$. gonorrhoeae, we repeated the experiment using IL-17RA-knockout (IL-17RA ${ }^{\mathrm{KO}}$ ) mice, ${ }^{9}$ which lack the principal receptor for IL-17A. In this experiment, C57BL/6 wild-type (WT) or IL-17RA ${ }^{\mathrm{KO}}$ mice were treated with estradiol and antibiotics, and then challenged with $5 \times 10^{6}$ colony-forming units (CFUs) and sampled for recoverable gonococci and neutrophil influx for the ensuing 7-10 days. WT control mice started to reduce the recoverable gonococcal load from day 4 and had cleared the infection by day 7 (Figure 6a, b). In contrast, IL-17RA ${ }^{\mathrm{KO}}$ mice began to reduce the gonococcal load on day 9 and took 12 days to clear the infection; the difference in persistence of infection was significant $(P<0.001)$. The neutrophil influx, which began on day 3 in control mice, was almost completely abrogated in IL-17RA ${ }^{\mathrm{KO}}$ mice (Figure 6c). These results indicate that 


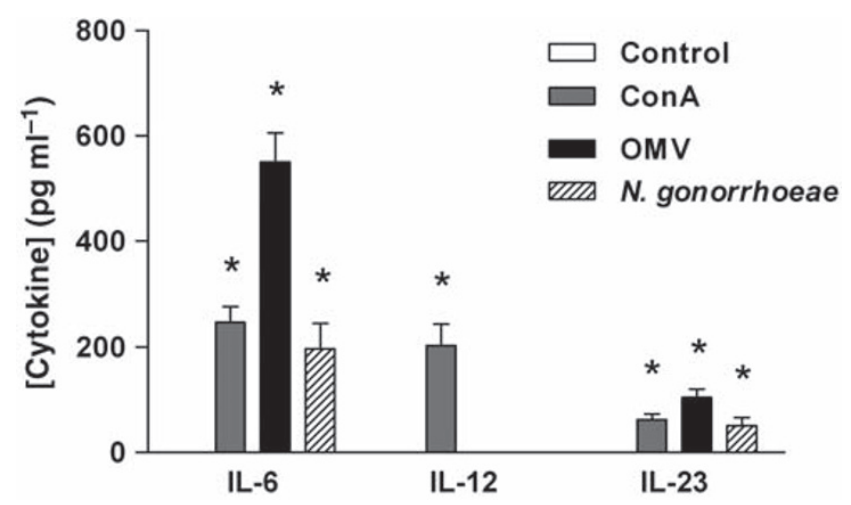

Figure 2 BMDCs produce IL-6 and IL-23, but not IL-12, in response to $N$. gonorrhoeae or its OMVs. BMDCs were incubated for $24 \mathrm{~h}$ in medium only (control) or with $2 \mu \mathrm{g} \mathrm{ml}^{-1}$ of ConA, N. gonorrhoeae at 25:1 $\mathrm{MOI}$, or $5 \mu \mathrm{g} \mathrm{ml}^{-1}$ of gonococcal OMVs. Supernatants were collected and assayed for cytokines by ELISA. All experiments were performed in triplicate and results are shown as mean \pm s.d.; ${ }^{*}$ indicates cytokine secretion significantly above control levels $(P<0.05$; Student's $t$-test). BMDC, bone marrow-derived dendritic cell; ConA, concanavalin-A; IL, interleukin; $\mathrm{MOI}$, multiplicity of infection; OMV, outer membrane vesicle.

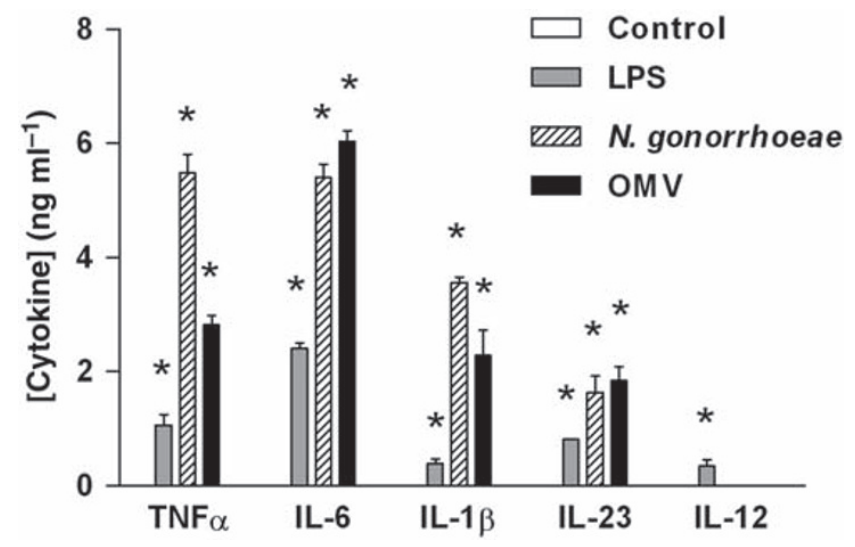

Figure 3 The human monocyte-like cell line THP-1 produces Th17associated cytokines in response to N. gonorrhoeae or its OMVs. Differentiated THP-1 cells were incubated for $24 \mathrm{~h}$ in medium only (control) or with $5 \mu \mathrm{g} \mathrm{ml}^{-1}$ of LPS, $5 \mu \mathrm{g} \mathrm{ml}^{-1}$ of gonococcal OMVs, or $N$. gonorrhoeae at an $\mathrm{MOI}$ of 25:1. Supernatants were assayed for cytokines by ELISA. All experiments were performed in triplicate and results are shown as mean \pm S.d.; * indicates cytokine secretion significantly above control levels $(P<0.01$; Student's $t$-test). LPS, lipopolysaccharide; $\mathrm{MOI}$, multiplicity of infection; OMV, outer membrane vesicle.

IL-17RA is important in neutrophil recruitment and clearance of N. gonorrhoeae in this mouse model.

\section{IL-17-mediated induction of cytokines and chemokines in the genital tract}

To examine the production of cytokines and chemokines by the mouse genital tract, vaginal explants were cultured in vitro with or without live N. gonorrhoeae. Supernatants were recovered after 3 days and assayed by ELISA. Consistent with the observed production of cytokines in draining lymph nodes after in vivo infection, IL-6, IL-22, and IL-17A were detected in the supernatants of WT vaginal tissue cultured with $N$. gonorrhoeae, but not in control (unstimulated) cultures (Figure 7). These

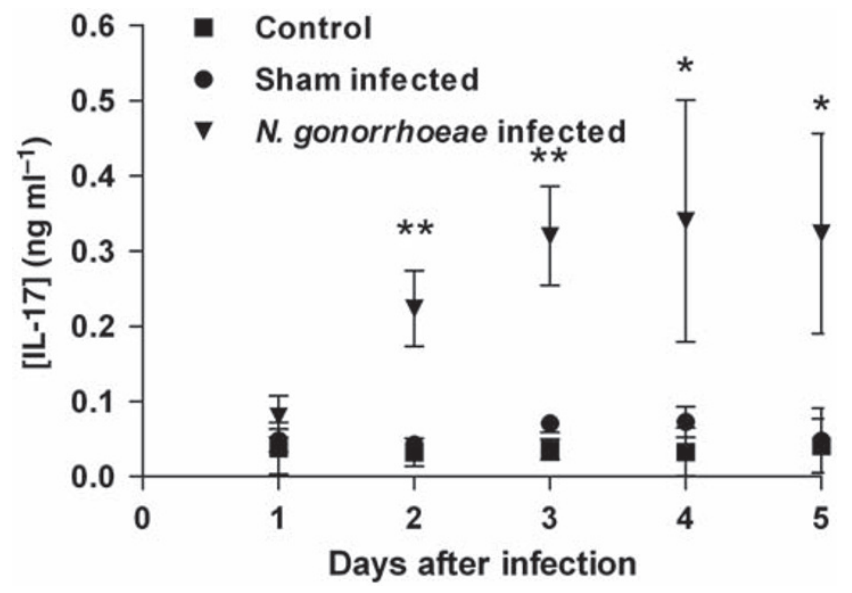

Figure 4 Production of IL-17 from draining iliac lymph node cells from mice infected with N. gonorrhoeae, or sham infected, or unmanipulated controls. Lymph nodes were collected on days 1-5 after infection and supernatants were assayed for IL-17 by ELISA after culture for $24 \mathrm{~h}$. Results are shown as mean \pm s.d. of triplicate cultures of lymph nodes pooled from three mice per time point. For days $2-5$, the difference between infected and sham-infected mice was statistically significant, ${ }^{* *} P<0.005,{ }^{\star} P<0.05$ (Student's $t$-test). IL, interleukin.

supernatants also contained the CXC chemokines KC, LIX, and MIP- $2 \alpha$ (Figure 7). The supernatants of vaginal explants from IL-17RA ${ }^{\mathrm{KO}}$ mice contained IL-17, IL-22, and $\mathrm{KC}$ at the same levels as in WT tissue, but showed diminished levels of IL-6, LIX, and MIP-2 $\alpha$ (Figure 7). Such diminished chemokine production was consistent with defective neutrophil recruitment seen in vivo in IL-17RA ${ }^{\mathrm{KO}}$ mice, or in those injected with IL-17A-blocking antibodies. Collectively, these experiments show that production of neutrophil-attractant chemokines in response to $N$. gonorrhoeae is at least partially IL-17-dependent.

\section{DISCUSSION}

Using a female mouse model of genital tract infection we have demonstrated a significant role for the newly described "Th17 axis of immunity" in response to $N$. gonorrhoeae. The evidence for this consists of the following: N. gonorrhoeae induces the in vitro production of the signature Th17 cytokine, IL-17A, as well as several other key cytokines of the Th17 pathway, in murine mononuclear cell cultures. These cytokines include IL-6, IL-1 $\beta$, and TNF- $\alpha$, which are known to be important for inducing the development of the Th17 lineage from naïve CD4+ T-cell precursors. ${ }^{12,13}$ The majority of IL-17-secreting cells were $\mathrm{CD} 4+\alpha \beta$ T-cells, but some $\gamma \delta$ T-cells also secreted IL-17A in response to $N$. gonorrhoeae. Notably we did not detect IL-12 production, either in spleen cell cultures, DC cultures, or genital tract explants. Previous reports of cytokine induction by N. gonorrhoeae failed to detect IL-12 (p70), ${ }^{30-32}$ which is well known to direct the development of Th1 cells. The related cytokine, IL-23, which shares the common p40 chain with IL-12, and is a key cytokine for the maintenance and functional differentiation of Th17 cells, ${ }^{12}$ was also not detected in the supernatants of spleen cells cultured with $N$. gonorrhoeae or in genital tract explants. However, when murine BMDCs were cultured with N. gonorrhoeae, production of IL-23 was observed, along with 

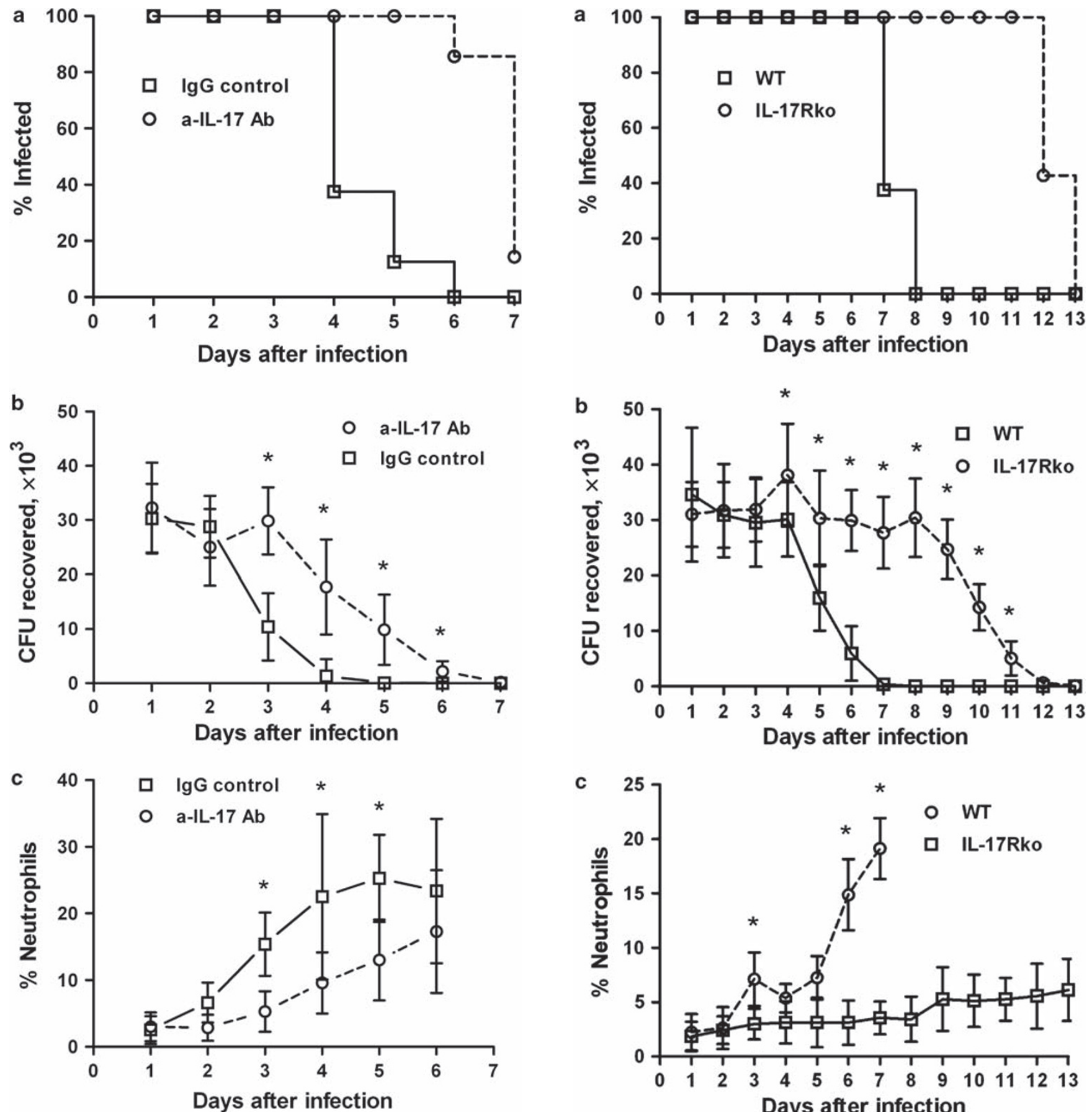

Figure 5 Blocking IL-17A prolongs gonococcal genital tract infection and delays the recruitment of neutrophils in mice. BALB/c mice were treated with anti-IL-17 antibody $(n=7)$ or rat $\operatorname{lgG}(n=8)$ and infected vaginally with $N$. gonorrhoeae. The course of infection was monitored by culture of vaginal mucus, and neutrophil influx was determined by microscopic examination of vaginal smears. (a) Percentage of mice infected with $N$. gonorrhoeae; the survival curves are significantly different $(P<0.001$; Kaplan-Meier). (b) Number of recoverable $N$. gonorrhoeae (CFUs), mean \pm s.d.; * indicates significant difference between treated and control groups ( $P<0.05$; Student's $t$-test).

(c) Neutrophil influx, measured as the percentage of neutrophils relative to other cells, mean \pm s.d.; * indicates significant difference between treated and control groups ( $P<0.05$; two-way analysis of variance). CFU, colony-forming unit; IL, interleukin.

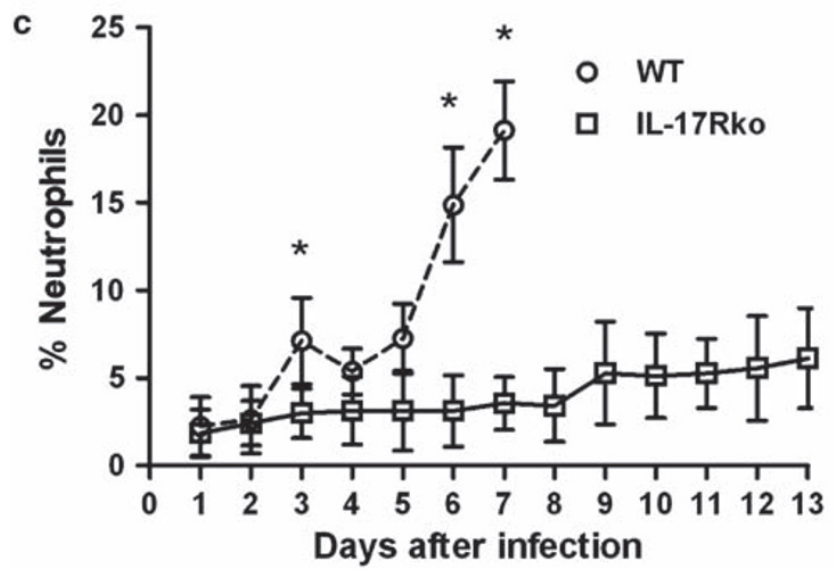

Figure 6 Prolongation of infection and suppression of neutrophil recruitment in IL-17RA ${ }^{\mathrm{KO}}$ mice. IL-17RA ${ }^{\mathrm{KO}}(n=7)$ or WT $(n=8) \mathrm{C} 57 \mathrm{BL} / 6$ mice were infected vaginally with $N$. gonorrhoeae. The course of infection was monitored by culture of vaginal mucus, and neutrophil influx was determined by microscopic examination of vaginal smears.

(a) Percentage of mice infected with $N$. gonorrhoeae; the survival curves are significantly different $(P<0.001$; Kaplan-Meier). (b) Number of recoverable N. gonorrhoeae (CFUs), mean \pm s.d.; * indicates significant difference between IL-17RA ${ }^{\mathrm{KO}}$ and WT groups $(P<0.05$; Student's $t$-test). (c) Neutrophil influx, measured as the percentage of neutrophils relative to other cells, mean \pm s.d.; ${ }^{*}$ indicates significant difference between IL-17RA ${ }^{\mathrm{KO}}$ and WT groups ( $P<0.05$; two-way analysis of variance). CFU, colony-forming unit; IL, interleukin; IL-17R, interleukin-17 receptor; KO, knockout; WT, wild type. 


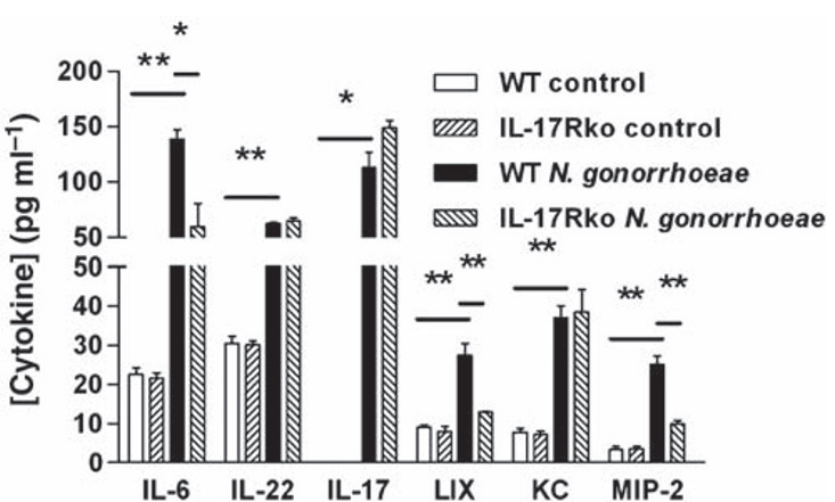

Figure 7 Production of cytokines and chemokines from mouse vaginal explants cultured with $N$. gonorrhoeae. Genital tract tissue segments from WT or IL-17RA ${ }^{\mathrm{KO}}$ mice were incubated in vitro in medium only (control) or with N. gonorrhoeae at a concentration of $1 \times 10^{7} \mathrm{CFUs} / \mathrm{ml}$. Supernatants were removed after 3 days and assayed for cytokines and chemokines by ELISA. The results are shown as mean \pm s.d. of triplicate cultures. Cytokine or chemokine production in the presence of $N$. gonorrhoeae was significantly higher than that in the corresponding controls or in IL-17RA ${ }^{\mathrm{KO}}$ cultures; ${ }^{* \star} P<0.01,{ }^{\star} P<0.05$ (Student's $t$-test). $\mathrm{CFU}$, colony-forming unit; IL, interleukin; IL-17R, interleukin-17 receptor; $\mathrm{KO}$, knockout; WT, wild type.

IL-6, but not IL-12. Importantly also, human THP-1-derived macrophages also produced IL-23 as well as IL- $1 \beta$, IL-6, and TNF- $\alpha$, but not IL-12, when stimulated with N. gonorrhoeae, implying that in this respect human and mouse APCs behave similarly in response to N. gonorrhoeae.

The components of $N$. gonorrhoeae that induce IL-17 appear to be predominantly heat-stable materials, including LOS, rather than proteins, and particularly not Opa proteins. Moreover, the in vitro response to gonococci was diminished in the absence of TLR4, but not in the absence of TLR2. However, the residual response of TLR4-deficient spleen cells to gonococcal cells or OMVs suggests that other components could be involved as well. Our ongoing analysis of the host response to N. gonorrhoeae during experimental infection of TLR4-deficient mice further supports a link between TLR4 signaling and IL-17 expression (M Packiam, SJ Veit, N Mavrogiorgos, RR Ingalls, and AE Jerse, unpublished data). Although different strains of mice vary in susceptibility to vaginal gonococcal infection, ${ }^{35}$ we observed no great differences between the production of IL-17A by spleen cells from BALB/c, C57BL/6, or C3H WT mice upon culture with N. gonorrhoeae.

To investigate the production and role of IL-17A in response to $N$. gonorrhoeae infection in vivo, we used the mouse model of lower genital tract infection. ${ }^{34}$ IL-17A was released into the culture supernatants of mononuclear cells from iliac lymph nodes, which drain the genital tract, collected from mice challenged with N. gonorrhoeae, but not sham-challenged mice. Production of IL-17A was observed in cells obtained from mice that been infected at least 2 days and continued for the duration of the infection. This finding suggests that Th17 cells constitute a component of the response to N. gonorrhoeae in vivo.

To further examine the significance of IL-17A production and its role in eliciting innate immune defenses, including the characteristic influx of neutrophils into the genital tract, in response to gonococcal infection, we used two approaches. First we used antibody-mediated blockade to inhibit the interaction of IL-17A with its main receptor, IL-17RA. Treatment of mice with monoclonal antibody to murine IL-17RA was only partially effective in inhibiting the neutrophil influx or altering the course of infection with $N$. gonorrhoeae (data not shown). However, treatment of mice with monoclonal antibody to murine IL-17A prolonged infection with $N$. gonorrhoeae by $2-3$ days concomitant with diminished influx of neutrophils, strongly suggesting that IL-17A-mediated responses are important for these outcomes. This conclusion was strengthened by experiments using IL-17RA ${ }^{\mathrm{KO}}$ mice, which lack the main receptor for IL-17A (reference Ye et al. ${ }^{9}$ ). These mice failed to show a measurable neutrophil influx in response to gonococcal challenge, and took 4-5 days longer to clear the infection than their WT counterparts. Despite the apparent lack of neutrophil influx, however, the IL-17RA ${ }^{\mathrm{KO}}$ mice eventually cleared the infection, suggesting that although phagocytosis of $N$. gonorrhoeae by neutrophils might contribute to their elimination, clearance was not absolutely dependent on neutrophils. It is likely that many factors are involved in the clearance of infection, or failure to sustain colonization, especially as mouse is not a natural host for N. gonorrhoeae.

Some insight into the mechanisms involved in IL-17A-dependent responses against $N$. gonorrhoeae was obtained from experiments using explants of murine genital tract cultured in vitro with $N$. gonorrhoeae. Assay of supernatants from these cultures revealed the release of not only IL-6, IL-17A, and IL-22, but also the CXC chemokines KC, LIX, and MIP-2, which are chemotactic for neutrophils. ${ }^{36}$ Notably, while production of IL-17A and IL-22 was unimpaired in the cultures of explants derived from IL-17RA ${ }^{\mathrm{KO}}$ mice, production of two of the chemokines, LIX and MIP-2, was diminished, suggesting their dependence on signaling through IL-17RA. Upregulation of CXC chemokines is a well-known effect of IL-17A on target IL-17RA-bearing cells; thus this finding is consistent with a key role for IL-17A. ${ }^{19}$

One of the acknowledged difficulties in investigating the pathogenesis of and immunity to $N$. gonorrhoeae is the lack of accessible animal models that reflect the human disease. Despite numerous efforts over several decades to devise alternatives to non-human primates, ${ }^{37}$ the only currently available model is the female mouse lower genital tract infection model. ${ }^{34}$ The infections persist for 5-12 days (or even longer in some cases ${ }^{38}$ ) depending on the strain of mouse and other factors. Bacterial replication and antigenic variation of Opa proteins occur during infection, and there is a host response, ${ }^{34,39}$ indicating that this is an infection model. Moreover, the mice usually show local neutrophil influx, as occurs in symptomatic human infection, thereby indicating inflammatory response by the mice. Whether or not neutrophils are responsible for phagocytic clearance of the infection, is debatable since it is known that $N$. gonorrhoeae can survive intracellular killing mechanisms within neutrophils. ${ }^{40-43}$ Although clearance appears to follow neutrophil influx, other mucosal defense mechanisms might contribute importantly to this. However, the nature of these defenses and the mechanisms involved in their recruitment, have not been understood, 
and numerous other factors undoubtedly limit the ability of N. gonorrhoeae to infect mice persistently. Furthermore, similar to humans, mice do not develop detectable antibody responses to vaginal gonococcal infection after either primary or secondary infection, and secondary infection is no more effectively resisted than primary infection. ${ }^{39}$ Thus mice afford an opportunity to study early in vivo responses of the immune system to gonococcal exposure.

Studies of immunity to gonorrhea have been fraught with difficulty over many decades. In large part this is due to the extraordinary antigenic variability of N. gonorrhoeae in which all the major surface components are subject to phase-variable on-off switching, genetic polymorphism, and recombinatorial expression. ${ }^{27,37}$ This variability has greatly complicated the evaluation of antibody responses in the serum and secretions of infected subjects. While some studies have revealed modest increases of serum antibodies to porin, the major outer membrane protein, and some evidence has been obtained for partial serovar-specific immunity in very highly exposed persons, ${ }^{44}$ the overall consensus from multiple studies, including sequential studies, is that specific adaptive immune responses to gonorrhea are minimal. ${ }^{28,45,46}$ Studies of cell-mediated immunity are few, although T-cell proliferation and cytokine secretion have been reported. ${ }^{28,47}$ However, it is well known that gonorrhea does not induce a state of protective immunity against future infection. ${ }^{26}$ Repeat infections are common, and they occur with no apparent diminution in severity, duration, or probability of acquisition from exposure. The conventional view is that $N$. gonorrhoeae can resist host responses by a combination of strategies including antigenic variation, which enables it to evade destruction by specific immune defenses, as well as several mechanisms for resisting complement activation and lysis. ${ }^{48-}$ ${ }^{50}$ An alternative view now emerging is that $N$. gonorrhoeae avoids inducing adaptive immune responses in the first place, and elicits instead innate immune responses that it is capable of resisting. In other words, like many pathogens that have become evolutionarily well-adapted to their hosts, $N$. gonorrhoeae induces host responses that are favorable to its survival. We propose that elicitation of IL-17 and other Th17-driven responses by $N$. gonorrhoeae is an example of this strategy. Th17 cells function as a bridge between adaptive and innate immunity, through production of the cytokines IL-17, IL-22, and others. ${ }^{13}$ IL-17Rs are widely distributed on epithelial, endothelial, and stromal cells, and ligation by IL-17A and/or IL-17F leads to signaling pathways that can result in the secretion of factors involved in innate defense mechanisms. ${ }^{13}$ These include the granulocyte-colonystimulating factor, which mobilizes neutrophils from the bone marrow reserves, and CXC chemokines KC, LIX, and MIP-2 in mice, or IL-8 in humans, which direct neutrophils to the site of microbial invasion and to some extent activate them. ${ }^{36}$ IL-17A and IL-22 act on mucosal epithelial cells to induce the secretion of several potent antimicrobial proteins, including $\beta$-defensins, S-100 proteins, RegIII $\gamma$, and lipocalin-2 (reference Ouyang et al. ${ }^{13}$ ). The effects of all these on N. gonorrhoeae are not yet known, but it can resist destruction by defensins in part through a multiple drug-exporter mechanism. ${ }^{51}$ In addition, intracellular residence within neutrophils ${ }^{43}$ where it can resist intracellular killing mechanisms, or within epithelial cells, ${ }^{52}$ may enable it to escape from soluble antimicrobial factors.

The diversion of host responses toward Th17 and away from Th1- or Th2-driven adaptive immunity may be one mechanism of evading the generation of specific immune responses, including antibodies that might, if directed to appropriate antigenic epitopes, be effective in destroying $N$. gonorrhoeae. Recent studies have implicated IL-17A in the suppression of Th1 cells..$^{53}$ In this context it is noteworthy that, both in our experiments and in several other reports, ${ }^{30-32} \mathrm{IL}-12$, a key cytokine in the induction of Th1 responses, was not observed. In addition, it is possible that inducible T-regulatory cells could form part of the host response pattern elicited by $N$. gonorrhoeae, as already suggested in the mouse model. ${ }^{54}$ It is noteworthy that inducible T-regulatory cells and Th17 lineages appear to be closely related in that both are driven by the key cytokine, transforming growth factor- $\beta$. Whereas transforming growth factor- $\beta$ acting alone programs $\mathrm{CD} 4+$ precursor T-cells to develop as inducible T-regulatory cells, transforming growth factor- $\beta$ in the presence of IL- 6 or IL-1 drives the development of Th17 cells. ${ }^{12}$ Conceivably therefore, a crucial factor in the nature of the host response to N. gonorrhoeae may be whether IL-6 or IL-1 is also generated by the responding APCs. In an earlier study of the cytokine responses of humans infected with gonorrhea, we observed the presence of IL- 6 and IL- 1 in the serum and secretions of some, but not all, infected patients. ${ }^{28}$ The significance of those findings for Th17 responses, which had not been described at that time, was not understood, and therefore cytokine responses in human subjects presenting with different clinical manifestations of gonococcal infection deserve re-evaluation, especially by exploiting newer and more sensitive technologies.

Overall the evidence from these studies of a murine model of gonococcal genital tract infection reveals that IL-17 and Th17 cells represent an entirely novel aspect of the host immuneinflammatory response to $N$. gonorrhoeae. If this applies also to the human infection, then it may represent a new paradigm in our understanding of this underappreciated, yet all too frequent human disease. Studies are in progress to address that important question.

\section{METHODS}

Mice. IL-17RA ${ }^{\mathrm{KO}}$ mice on a C57BL/6 background were provided by Amgen (Seattle, WA). WT mice (C57BL/6 and BALB/c) were obtained from Harlan Sprague Dawley (Indianapolis, IN). C3H/HeJ and C3H/ $\mathrm{FeJ}$ mice were purchased from Jackson Laboratories (Bar Harbor, ME). TLR2-knockout mice (C57BL/6 background) were provided by Dr Terry Connell (University at Buffalo). The mice were kept in HEPAfiltered cages with autoclaved food, water, and bedding. For each experiment, the animals used were age- and strain-matched females between $8-10$ weeks of age. All procedures were performed in accordance with protocols approved by the University at Buffalo Institutional Animal Care and Use Committee, and in accordance with Federal and State regulations governing animal welfare. 
Bacteria. N. gonorrhoeae FA1090 (streptomycin-resistant, serumresistant), ${ }^{55}$ provided by Dr Janne Cannon (University of North Carolina at Chapel Hill), was used in most experiments. N. gonorrhoeae strain PID2 and its purified LOS were obtained from Dr Gary Jarvis (University of California San Francisco). The bacteria were cultured on GC agar supplemented with hemoglobin and Isovitalex (BD Biosciences, San Jose, CA) under an atmosphere of $5 \% \mathrm{CO}_{2}$ at $37^{\circ} \mathrm{C}$ and the resultant growth was checked for colony morphology consistent with Opa protein and pilus expression. Bacteria were harvested from plates using a sterile inoculating loop after $18-24 \mathrm{~h}$ of growth and suspended in phosphate-buffered saline. Bacterial cell density was determined by measuring the optical density at $600 \mathrm{~nm}$ and reference to a previously determined calibration curve.

Gonococcal OMVs. N. gonorrhoeae was grown on chocolate agar plates under an atmosphere of $5 \% \mathrm{CO}_{2}$ at $37^{\circ} \mathrm{C}$ overnight. Colony morphology was examined to confirm Opa protein and pilus expression. Bacteria were harvested from plates into ice-cold lithium acetate buffer ( $\mathrm{pH}$ 5.8) and passed through a $25-\mathrm{G}$ needle 10-12 times to sheer the outer membranes from the bacteria. The suspensions were spun in microfuge tubes at 13,000 r.p.m. for $1 \mathrm{~min}$. The supernatants were collected and ultracentrifuged at $107,000 \times g$ for $2 \mathrm{~h}$. The pellet was collected and resuspended in $50 \mathrm{mM}$ Tris- $\mathrm{HCl}$ at $\mathrm{pH} 8.0$.

Splenic cells. Mouse mononuclear cells were isolated from aseptically harvested spleens using Histopaque 1083 (Sigma, St Louis, MO) density-gradient centrifugation. The number of viable cells obtained was generally over $90 \%$ as determined by trypan blue (Invitrogen, Carlsbad, CA) dye exclusion cell counts. Cells were cultured in 6- or 24-well culture plates at a cell density of $2 \times 10^{6} \mathrm{cells} \mathrm{ml}^{-1}$ with either no stimulus, $2 \mu \mathrm{g} \mathrm{ml}^{-1}$ of the mitogen ConA, $5 \mu \mathrm{g} \mathrm{ml}^{-1}$ of $N$. gonorrhoeae OMVs, or live N. gonorrhoeae cells at a multiplicity of infection (MOI) of 10:1. Supernatants were collected from the cell cultures after 1-5 days and assayed for IL- $1 \beta$, TNF $\alpha$, IL-12, IL-17, IL-6, IFN- $\gamma$, and IL- 22 by ELISA using kits from eBioscience (San Diego, CA) and BD Biosciences.

Bone marrow-derived DCs. Mouse femurs and tibias were removed and muscle was removed from the bones. The bones were immersed in 70\% ethanol for $2 \mathrm{~min}$ and washed with cold RPMI-1640. Both epiphyses of each bone were cut off and the marrow was flushed out of the shafts with RPMI. Red blood cells were removed by lysing with ammonium chloride. Viable cells were suspended at a concentration of $1 \times 10^{6} \mathrm{cells} \mathrm{ml}^{-1}$ and cultured in the presence of $1 \mathrm{ng} \mathrm{ml}^{-1}$ of murine granulocyte-macrophage-colony-stimulating factor. The cells were washed and fed every 2 days and microscopically monitored for the formation of dendrites and adherence to the culture wells, which is consistent with the development of DCs. After 1 week of culture, either E. coli lipopolysaccharide as a positive control or N. gonorrhoeae OMVs was added to the cultures; control cultures were continued in medium only. The cells were then incubated for $24 \mathrm{~h}$ and supernatants were harvested and assayed for production of IL-12, IL-23, and IL-6.

THP-1 cells. Human monocyte-like THP-1 cells (ATCC TIB-202) were cultured in RPMI-1640 supplemented with $10 \%$ heat-inactivated fetal bovine serum, $2 \mathrm{~mm}$ L-glutamine, $10 \mathrm{~mm}$ HEPES, $100 \mathrm{U} \mathrm{ml}^{-1}$ penicillinG, $100 \mu \mathrm{g} \mathrm{ml}^{-1}$ streptomycin, and $0.05 \mathrm{~mm} 2$-mercapthoethanol. THP-1 cells $\left(1.5 \times 10^{5}\right.$ cells per well $)$ were differentiated for 3 days in the presence of $10 \mathrm{ng} \mathrm{ml}^{-1}$ phorbol myristate acetate. The cells were then incubated with either no additional stimulus, $5 \mu \mathrm{g} \mathrm{ml}^{-1}$ E. coli lipopolysaccharide (Sigma), $5 \mu \mathrm{g} \mathrm{ml}^{-1}$ gonococcal OMVs, or N. gonorrhoeae at an MOI of 25:1. Supernatants were removed after $24 \mathrm{~h}$ and assayed for production of IL-6, TNF $\alpha$, IL-1 $\beta$, and IL-23 by ELISA (eBioscience).

Murine vaginal infection model. Female mice between 8 and 10 weeks old were infected with live $N$. gonorrhoeae as previously described by Jerse, ${ }^{34}$ with the modification that water-soluble estradiol was used as described by Song et al. ${ }^{39}$ Briefly, groups of 10-12 mice in diestrus or anestrus were identified by examination of stained vaginal smears and injected subcutaneously with $500 \mu \mathrm{g}$ of $17 \beta$-estradiol 2 days before infection (day -2$)$; estradiol treatment was repeated on days 0 and 2 . To reduce the overgrowth of flora that occurs under the influence of estradiol, mice were injected intraperitoneally with streptomycin $(0.6 \mathrm{mg})$ and vancomycin $(3.6 \mathrm{mg})$ twice daily for the duration of the experiment, and trimethoprim sulfate $\left(0.4 \mathrm{mg} \mathrm{ml}^{-1}\right)$ was added to the drinking water. For IL-17-blockade experiments, mice were injected on days $-1,0$, and every 2 days thereafter with $70 \mu \mathrm{g}$ of either rat monoclonal IgG2a anti-IL-17-blocking antibody (M210; provided by Amgen) or rat IgG control (Caltag, Burlingame, CA). On day 0 mice were infected with $2 \times 10^{6} \mathrm{CFUs}$ of $N$. gonorrhoeae freshly harvested after growth for $18 \mathrm{~h}$ and suspended in $20 \mu \mathrm{l}$ of phosphatebuffered saline. Bacterial colonization loads were determined daily by collecting vaginal mucus with a sterile swab and suspending it in $100 \mu \mathrm{l}$ of phosphate-buffered saline, followed by serial dilution and culture on chocolate agar containing the selective antibiotics vancomycin, colistin, nystatin, trimethoprim sulfate, and streptomycin (VCNTS). The plates were incubated overnight in $5 \% \mathrm{CO}_{2} /$ air at $37^{\circ} \mathrm{C}$ and colonies were counted. Commensal bacteria were monitored by culturing a portion of the swab contents on brain-heart infusion agar. Vaginal mucus was also smeared onto glass slides, stained with Hema 3 staining solution (Fisher Scientific, Kalamazoo, MI), and the number of neutrophils relative to 100 vaginal epithelial cells was counted. Mice that developed an overgrowth of commensal flora were eliminated, leaving at least seven animals per group to complete the experiment.

Generation of IL-17 in vivo. Mice were either left untreated, mock infected with vehicle only, or infected with $N$. gonorrhoeae. On days 1 through 5 , mice were killed and draining iliac lymph nodes were excised and teased apart to release the cells, which were then passed through a cell strainer and washed. Approximately $2 \times 10^{6} \mathrm{cells} \mathrm{ml}^{-1}$ were cultured for $24 \mathrm{~h}$ under $5 \% \mathrm{CO}_{2} /$ air at $37^{\circ} \mathrm{C}$. Supernatants were removed and assayed for IL-17 and other cytokines using ELISA kits (eBioscience).

Vaginal explants. Mouse genital tracts were dissected out aseptically and washed with Hanks' buffered salt solution. The tissue was manually separated into 1- to 2-mm pieces, washed again, and weighed. Equal weights were added to each well of the cell culture plates. Tissue was grown under $5 \% \mathrm{CO}_{2}$ /air at $37^{\circ} \mathrm{C}$ in RPMI- 1640 supplemented with $10 \%$ heat-inactivated fetal bovine serum, $2 \mathrm{~mm} \mathrm{~L}$-glutamine, $10 \mathrm{~mm}$ HEPES, $100 \mathrm{U} \mathrm{ml}^{-1}$ penicillin-G, $100 \mu \mathrm{g} \mathrm{ml}^{-1}$ streptomycin, and $1 \mu \mathrm{g} \mathrm{ml}^{-1}$ fungizone. The explants were cultured for 3 days with $2 \times 10^{7} \mathrm{~N}$. gonorrhoeae per milliliter or $2 \mu \mathrm{g} \mathrm{ml}^{-1}$ of ConA, or in medium only (controls). Supernatants were removed and assayed for IL-6, IL-17A, IL-22, LIX, KC, and MIP-2 $\alpha$ by ELISA using reagents and kits obtained from eBioscience or R\&D Systems (Minneapolis, MN).

Statistics. Statistical analysis was performed using Prism 5 (GraphPad Software, San Diego, CA). Student's $t$-test or two-way analysis of variance with Bonferroni post hoc test was used to determine the significance of difference of means, and Kaplan-Meier survival analysis was used to evaluate the persistence of infection in mice, using the log-rank test. $P<0.05$ (two-tailed) was considered significant.

\section{ACKNOWLEDGMENTS}

We thank Dawn Both-Kim for technical assistance and Amgen for providing IL-17RA ${ }^{\mathrm{KO}}$ mice and antibodies to IL-17 and IL-17R. Dr Gary Jarvis (University of California San Francisco) provided the gonococcal LOS and the corresponding strain of $N$. gonorrhoeae, and Dr Terry Connell (University at Buffalo) provided TLR2-knockout mice. This study was supported by a grant from the John R Oishei Foundation, Buffalo, NY (to MWR), and grants Al074791 (to MWR), U19 Al31496 (to AEJ), and AR054389 and DE018822 (to SLG), from the National Institutes of Health. B.F. was supported by the University at Buffalo School of Medicine and Biomedical Sciences. 


\section{DISCLOSURE}

The authors declared no conflict of interest.

(C) 2010 Society for Mucosal Immunology

\section{REFERENCES}

1. Harrington, L.E. et al. Interleukin 17-producing CD4+ effector T cells develop via a lineage distinct from the Thelper type 1 and 2 lineages. Nat. Immunol. 6, 1123-1132 (2005).

2. Park, $\mathrm{H}$. et al. A distinct lineage of CD4T cells regulates tissue inflammation by producing interleukin 17. Nat. Immunol. 6, 1133-1141 (2005).

3. Kotake, S. et al. IL-17 in synovial fluids from patients with rheumatoid arthritis is a potent stimulator of osteoclastogenesis. J. Clin. Invest. 103, 1345-1352 (1999)

4. Langrish, C.L. et al. IL-23 drives a pathogenic T cell population that induces autoimmune inflammation. J. Exp. Med. 201, 233-240 (2005).

5. Happel, K.I. et al. Divergent roles of IL-23 and IL-12 in host defense against Klebsiella pneumoniae. J. Exp. Med. 202, 761-769 (2005).

6. Higgins, S.C., Jarnicki, A.G., Lavelle, E.C. \& Mills, K.H. TLR4 mediates vaccine-induced protective cellular immunity to Bordetella pertussis: role of IL-17-producing T cells. J. Immunol. 177, 7980-7989 (2006).

7. Khader, S.A. et al. IL-23 and IL-17 in the establishment of protective pulmonary CD4+ T cell responses after vaccination and during Mycobacterium tuberculosis challenge. Nat. Immunol. 8, 369-377 (2007)

8. Shibata, K., Yamada, H., Hara, H., Kishihara, K. \& Yoshikai, Y. Resident $\vee \delta 1+\gamma \delta T$ cells control early infiltration of neutrophils after Escherichia coli infection via IL-17 production. J. Immunol. 178, 4466-4472 (2007).

9. Ye, P. et al. Requirement of interleukin 17 receptor signaling for lung CXC chemokine and granulocyte colony-stimulating factor expression, neutrophil recruitment, and host defense. J. Exp. Med. 194, 519-527 (2001).

10. Yu, J.J. et al. An essential role for IL-17 in preventing pathogen-initiated bone destruction: recruitment of neutrophils to inflamed bone requires IL-17 receptor-dependent signals. Blood 109, 3794-3802 (2007)

11. Weaver, C.T., Harrington, L.E., Mangan, P.R., Gavrieli, M. \& Murphy, K.M. Th17: an effector CD4T cell lineage with regulatory T cell ties. Immunity 24, 677-688 (2006).

12. Korn, T., Bettelli, E., Oukka, M. \& Kuchroo, V.K. IL-17 and Th17 Cells. Annu. Rev. Immunol. 27, 485-517 (2009).

13. Ouyang, W., Kolls, J.K. \& Zheng, Y. The biological functions of Thelper 17 cell effector cytokines in inflammation. Immunity 28, 454-467 (2008).

14. LeibundGut-Landmann, S. et al. Syk- and CARD9-dependent coupling of innate immunity to the induction of $T$ helper cells that produce interleukin 17. Nat. Immunol. 8, 630-638 (2007).

15. Verreck, F.A. et al. Human IL-23-producing type 1 macrophages promote but IL-10-producing type 2 macrophages subvert immunity to (myco)bacteria. Proc. Natl. Acad. Sci. USA 101, 4560-4565 (2004).

16. Schulz, S.M. et al. Protective immunity to systemic infection with attenuated Salmonella enterica serovar Enteritidis in the absence of IL-12 is associated with IL-23-dependent IL-22, but not IL-17. J. Immunol. 181 7891-7901 (2008)

17. Prause, O., Laan, M., Lotvall, J. \& Linden, A. Pharmacological modulation of interleukin-17-induced GCP-2-, GRO-alpha- and interleukin-8 release in human bronchial epithelial cells. Eur. J. Pharmacol. 462, 193-198 (2003).

18. Ruddy, M.J., Shen, F., Smith, J.B., Sharma, A. \& Gaffen, S.L. Interleukin17 regulates expression of the CXC chemokine LIX/CXCL5 in osteoblasts: implications for inflammation and neutrophil recruitment. J. Leuk. Biol. 76, 135-144 (2004).

19. Shen, F., Ruddy, M.J., Plamondon, P. \& Gaffen, S.L. Cytokines link osteoblasts and inflammation: microarray analysis of interleukin-17- and TNF- $\alpha$-induced genes in bone cells. J. Leuk. Biol. 77, 388-399 (2005)

20. Witowski, J. et al. IL-17 stimulates intraperitoneal neutrophil infiltration through the release of GRO $\alpha$ chemokine from mesothelial cells. J. Immunol. 165, 5814-5821 (2000).

21. Huang, W., Na, L., Fidel, P.L. \& Schwarzenberger, P. Requirement of interleukin-17A for systemic anti-Candida albicans host defense in mice. J. Infect. Dis. 190, 624-631 (2004).

22. Conti, H.R. et al. Th17 cells and IL-17 receptor signaling are essential for mucosal host defense against oral candidiasis. J. Exp. Med. 206, 299-311 (2009).
23. Liang, S.C. et al. Interleukin (IL)-22 and IL-17 are coexpressed by Th17 cells and cooperatively enhance expression of antimicrobial peptides. J. Exp. Med. 203, 2271-2279 (2006).

24. Global prevalence and incidence of selected curable sexually transmitted infections: overview and estimates. World Health Organization (2001).

25. Notifiable diseases/deaths in selected cities weekly information. MMWR 55, 1396-140 (2009).

26. Noble, R.C., Kirk, N.M., Slagel, W.A., Vance, B.J. \& Somes, G.W. Recidivism among patients with gonococcal infection presenting to a venereal disease clinic. Sex Transm. Dis. 4, 39-43 (1977).

27. Edwards, J.L. \& Apicella, M.A. The molecular mechanisms used by Neisseria gonorrhoeae to initiate infection differ between men and women. Clin. Microbiol. Rev. 17, 965-981 (2004).

28. Hedges, S.R., Sibley, D.A., Mayo, M.S., Hook, E.W. 3rd \& Russell, M.W. Cytokine and antibody responses in women infected with Neisseria gonorrhoeae: effects of concomitant infections. J. Infect. Dis. 178, 742-751 (1998).

29. Fichorova, R.N., Desai, P.J., Gibson, F.C. 3rd \& Genco, C.A. Distinct proinflammatory host responses to Neisseria gonorrhoeae infection in immortalized human cervical and vaginal epithelial cells. Infect. Immun. $69,5840-5848$ (2001)

30. Naumann, M., Wessler, S., Bartsch, C., Wieland, B. \& Meyer, T.F. Neisseria gonorrhoeae epithelial cell interaction leads to the activation of the transcription factors nuclear factor $\kappa \mathrm{B}$ and activator protein 1 and the induction of inflammatory cytokines. J. Exp. Med. 186, 247-258 (1997).

31. Ramsey, K.H. et al. Inflammatory cytokines produced in response to experimental human gonorrhea. J. Infect. Dis. 172, 186-191 (1995).

32. Simpson, S.D., Ho, Y., Rice, P.A. \& Wetzler, L.M. T lymphocyte response to Neisseria gonorrhoeae porin in individuals with mucosal gonococcal infections. J. Infect. Dis. 180, 762-773 (1999).

33. Jerse, A.E. et al. Multiple gonococcal opacity proteins are expressed during experimental urethral infection in the male. J. Exp. Med. 179, 911-920 (1994).

34. Jerse, A.E. Experimental gonococcal genital tract infection and opacity protein expression in estradiol-treated mice. Infect. Immun. 67 5699-5708 (1999)

35. Packiam, M., Veit, S.J., Anderson, D.J., Ingalls, R.R. \& Jerse, A.E. Mouse strain-dependent differences in susceptibility to Neisseria gonorrhoeae infection and induction of innate immune responses. Infect. Immun. 78, 433-440 (2010).

36. Kobayashi, Y. The role of chemokines in neutrophil biology. Front Biosci. 13, 2400-2407 (2008)

37. Russell, M.W. \& Hook, E.W. Gonorrhea. In Vaccines for Biodefense and Emerging and Neglected Diseases (Barrett, A.D.T., and Stanberry, L.R., eds) 963-981 (Academic Press, London, 2009).

38. Jerse, A.E. et al. Growth of Neisseria gonorrhoeae in the female mouse genital tract does not require the gonococcal transferrin or hemoglobin receptors and may be enhanced by commensal lactobacilli. Infect. Immun. 70, 2549-2558 (2002).

39. Song, W. et al. Local and humoral immune responses against primary and repeat Neisseria gonorrhoeae genital tract infections of $17 \beta$ estradiol-treated mice. Vaccine 26, 5741-5751 (2008).

40. Criss, A.K., Katz, B.Z. \& Seifert, H.S. Resistance of Neisseria gonorrhoeae to non-oxidative killing by adherent human polymorphonuclear leucocytes. Cell. Microbiol. 11, 1074-1087 (2009).

41. Wu, H., Soler-Garcia, A.A. \& Jerse, A.E. A strain-specific catalase mutation and mutation of the metal-binding transporter gene mntC attenuate Neisseria gonorrhoeae in vivo but not by increasing susceptibility to oxidative killing by phagocytes. Infect. Immun. 77, 1091-1102 (2009)

42. Simons, M.P., Nauseef, W.M. \& Apicella, M.A. Interactions of Neisseria gonorrhoeae with adherent polymorphonuclear leukocytes. Infect. Immun. 73, 1971-1977 (2005).

43. Casey, S.G., Veale, D.R. \& Smith, H. Demonstration of intracellular growth of gonococci in human phagocytes using spectinomycin to kill extracellular organisms. J. Gen. Microbiol. 113, 395-398 (1979).

44. Plummer, F.A. et al. Epidemiologic evidence for the development of serovar-specific immunity after gonococcal infection. J. Clin. Invest. 83, 1472-1476 (1989).

45. Schmidt, K.A. et al. Experimental gonococcal urethritis and reinfection with homologous gonococci in male volunteers. Sex Transm. Dis. 28, 555-564 (2001). 
46. Price, G.A., Hobbs, M.M. \& Cornelissen, C.N. Immunogenicity of gonococcal transferrin binding proteins during natural infections. Infect. Immun. 72, 277-283 (2004).

47. Hedges, S.R., Mayo, M.S., Mestecky, J., Hook, E.W. 3rd \& Russell, M.W. Limited local and systemic antibody responses to Neisseria gonorrhoeae during uncomplicated genital infections. Infect. Immun. 67, 3937-3946 (1999).

48. Ram, S. et al. Binding of C4b-binding protein to porin: a molecular mechanism of serum resistance of Neisseria gonorrhoeae. J. Exp. Med. 193, 281-295 (2001).

49. Smith, H., Parsons, N.J. \& Cole, J.A. Sialylation of neisserial lipopolysaccharide: a major influence on pathogenicity. Microb. Pathog. 19, 365-377 (1995).

50. Rice, P.A., Vayo, H.E., Tam, M.R. \& Blake, M.S. Immunoglobulin G antibodies directed against protein III block killing of serum-resistant Neisseria gonorrhoeae by immune serum. J. Exp. Med. 164, 1735-1748 (1986).
51. Long, F., Rouquette-Loughlin, C., Shafer, W.M. \& Yu, E.W. Functional cloning and characterization of the multidrug efflux pumps NorM from Neisseria gonorrhoeae and YdhE from Escherichia coli. Antimicrob Agents Chemother 52, 3052-3060 (2008).

52. Nassif, X. \& So, M. Interaction of pathogenic neisseriae with nonphagocytic cells. Clin. Microbiol. Rev. 8, 376-388 (1995).

53. Awasthi , A.K.V. IL-17A directly inhibits TH1 cells and thereby suppresses development of intestinal inflammation. Nat. Immunol. 10, 568-570 (2009).

54. Imarai, M. et al. Regulatory T cells are locally induced during intravaginal infection of mice with Neisseria gonorrhoeae. Infect. Immun. 76, 5456-5465 (2008).

55. Cohen, M.S. et al. Human experimentation with Neisseria gonorrhoeae: rationale, methods, and implications for the biology of infection and vaccine development. J. Infect. Dis. 169, 532-537 (1994). 